\title{
Interpretation of Nuclear Quadrupole Constants in Compounds of ${ }^{181} \mathrm{Ta}$
}

Cite as: J. Chem. Phys. 50, 5040 (1969); https://doi.org/10.1063/1.1671009

Submitted: 02 December 1968 . Published Online: 19 December 2003

P. da R. Andrade
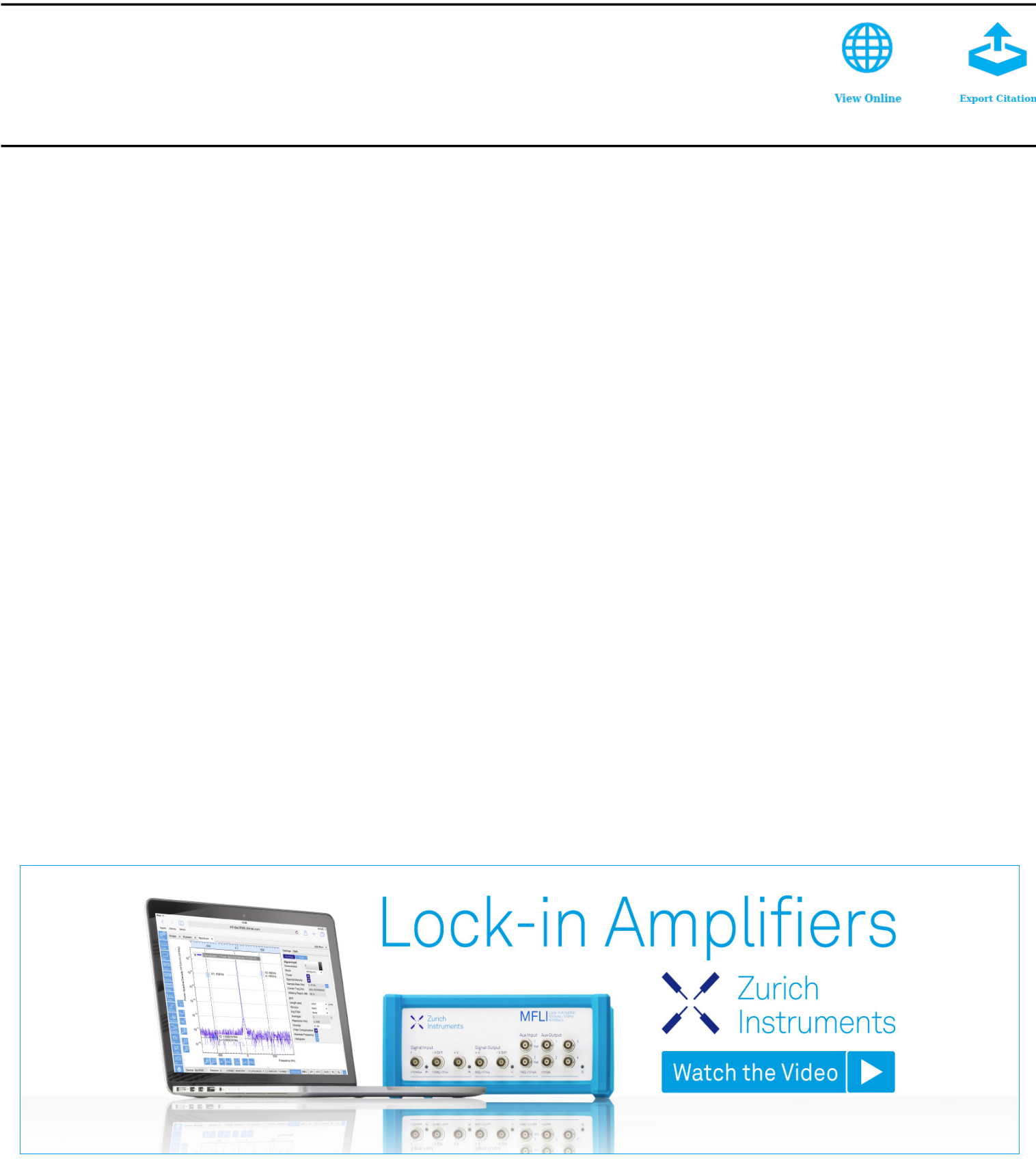
pected that charge transfer is more likely the longer the duration of the collision.

It is possible that a detailed reaction theory could predict the temperature dependence quantitatively for one or more statistical models and thus bear on unimolecular decomposition theory in a new manner.

${ }^{1}$ D. B. Dunkin, F. C. Fehsenfeld, A. L. Schmeltekopf, and E. E. Ferguson, J. Chem. Phys, 49, 1365 (1968).

${ }^{2}$ C. F. Giese, Advan. Chem. Ser. 58, 20 (1966).

${ }^{3}$ R. F. Stebbings, J. A. Rutherford, and B. R. Turner, J. Geophys. Res. 71, 771 (1966).

${ }_{4}$ D. K. Bohme, D. B. Dunkin, F. C. Fehsenfeld, and E. E. Ferguson, J. Chem. Phys. 49, 5201 (1968). Also E. E. Ferguson, D. B. Dunkin, F. C. Fehsenfeld, and A. L. Schmeltekopf, Bull. Am. Phys. Soc. 13, 212 (1968).

${ }^{6} \mathrm{H}$. S. Johnston, Gas Phase Reaction Rate Theory (Ronald Press Co., New York, 1966), Chap. 15.

'J. C. Light, J. Chem. Phys. 40, 3221 (1964); P. Pechukas and J. C. Light, ibid. 42, 3281 (1965).

\section{Interpretation of Nuclear Quadrupole Constants in Compounds of ${ }^{181} \mathrm{Ta}$}

\author{
P. DA R, ANDRADF \\ Instituto de Fisica, Universidade Federal do Rio Grande do Sul, \\ Pôrto Alegre-RS-Brasil*
}

(Received 2 December 1968)

Recently some nuclear quadrupole constants in compounds of ${ }^{181} \mathrm{Ta}$ of low oxidation states have been measured ${ }^{1-3}$ by several groups studying the perturbed angular correlation in the cascade $\left(\frac{1}{2}\right) 133 \mathrm{keV}\left(\frac{5}{2}\right) 482$ $\mathrm{keV}\left(\frac{7}{2}\right)$, where the intermediate state has a lifetime $\tau_{N}=15.8$ nsec. The compounds used were $\mathrm{HfO}_{2}$, $\mathrm{HfOCl}_{2} \cdot 8 \mathrm{H}_{2} \mathrm{O}$, and $\mathrm{Hf}$ metal. From the known nuclear quadrupole moment $(Q)$ of ${ }^{181} \mathrm{Ta}$ [using the formula $\left.\left|V_{z z}\right|=2 I(2 I+1) \hbar \omega_{0} / 3 e Q\right]$ electric field gradients (efg) produced by the atomic or molecular environment of the decaying nucleus were derived.

In this Note we have interpreted these electric field gradients in terms of the ligand-field theory ${ }^{4}$ which is mainly concerned with the effect of electrostatic potentials of different symmetries on electronic wavefunctions. Neglecting effects of hybridization, we can write a theoretical expression for the efg:

$$
V_{z z}=\sum_{n l m}\left|a_{n l m}\right|^{2} q_{n l m, n l m},
$$

where

$$
q_{n l m, n l m}=-e\left(\frac{16}{6}\right)^{1 / 2} \int \psi_{n l m} Y_{2}^{0} \psi_{n l m} * d z
$$

are the values of the efg for each atomic state. The $\psi_{n l m}$ 's are the wavefunctions of the ground state of the electronic configuration.

The nucleus ${ }^{181} \mathrm{Ta}$ originates from the decay of ${ }^{181} \mathrm{Hf}$; we shall assume that the electronic rearrangement following the decay occurs sufficiently fast so as to ensure that the experimentally measured quadrupole constants refer to the Ta compound structure.

From atomic spectroscopy, we known that the ground term for ${ }^{181} \mathrm{Ta}$ is ${ }^{4} F_{3 / 2}$ and the configuration of the outer electrons is $\left(5 d^{3}\right)\left(6 s^{2}\right)$. As closed orbits and $s$ wavefunctions do not contribute to the efg we need only to take into account the terms with $n=5, l=2$, in the given expression.

The electronic wavefunctions for a single $5 d$ electron and for three $5 d$ electrons may be obtained from Ref. 5 , and following Ref. 4 one can find the effect of an electrostatic potential on ground terms ${ }^{4} \mathrm{~F}$ for different local crystalline structures of the environment of the central atom (in our case, the atom of ${ }^{181} \mathrm{Ta}$ ). An electrostatic potential produced by ions forming an octahedron, a tetrahedron or a cube splits the energy levels for $d$ functions. This splitting for electron configurations $d^{1}$ and $d^{3}$ is also given in Ref. 4. For $d^{1}$, there is a splitting in two orbital sets $d \epsilon$ (threefold orbitally degenerate) and $d \gamma$ (twofold orbitally degenerate). For $d^{3}$, there is a splitting in three orbital sets $T_{1}$ (threefold orbitally degenerate), $T_{2}$ (threefold orbitally degenerate), and $A_{2}$ (not orbitally degenerate). Distortions of cubic symmetries are treated as perturbations. Their main effect is to lift the orbital degeneracy, leaving an electronic ground state.

With this theoretical background, it is possible to analyze the efg given in Table I:

(a) The crystalline structure of $\mathrm{TaO}_{2}$ has cubic symmetry ${ }^{6}$ and its electron configuration is $d^{\mathrm{I}}$. A cubic symmetry leaves a doubly degenerate ground term $E$ but a tetragonal distortion lifts the orbital degeneracy, resulting in a ground state $\left|x^{2}-y^{2}\right\rangle$.

(b) The local crystalline structure of Ta in $\mathrm{HfOCl}_{2}$. $8 \mathrm{H}_{2} \mathrm{O}$ has tetragonal symmetry. ${ }^{6}$ Its electron configura-

TABLE I. Comparison between experimental derived and theoretical estimated electric field gradients.

\begin{tabular}{lccccc}
\hline \hline Compound & $\begin{array}{c}\text { Electron } \\
\text { configuration } \\
\text { for Ta }\end{array}$ & $\begin{array}{c}\text { Ground } \\
\text { wavefunctions }\end{array}$ & $\begin{array}{c}q \text { in units } \\
\left\langle r^{-a}\right\rangle_{a-5}\end{array}$ & $\begin{array}{c}\left|V_{\text {as }}\right| \\
10^{17} \mathrm{~V} / \mathrm{cm}^{2}\end{array}$ & Reference \\
\hline $\mathrm{Hf}$ metal & $d^{3}$ & $\langle 0\rangle$ & $\frac{2}{5}(4 / 7)$ & $5.2 \pm 0.3$ & 1 \\
$\mathrm{HfO}_{2}$ & $d^{1}$ & $\left|x^{2}-y^{2}\right\rangle$ & $4 / 7$ & $13.5 \pm 1.2$ & 2 \\
$\mathrm{HfOCl}_{2} \cdot 8 \mathrm{H}_{2} \mathrm{O}$ & $d^{1}$ & $\left|x^{2}-y^{2}\right\rangle$ & $4 / 7$ & $13.3 \pm 1.2$ & 3 \\
\hline
\end{tabular}


tion is also $d^{1}$. It follows that we can assume for the ground state the form $\left|x^{2}-y^{2}\right\rangle$.

(c) The crystalline structure of metallic $\mathrm{Ta}$ is of cubic symmetry (bcc). Its electron configuration is $d^{3}$. The effect of an electrostatic potential due to a cubic charge distribution is to split the energy level leaving $T_{1}$ as the ground state. A tetragonal distortion lifts this threefold orbital degeneracy resulting ${ }^{4}$ in a ground state $\langle 0\rangle$.

Based on these assumptions, the values for $q_{n l m}$ in units of $\left\langle r^{-3}\right\rangle_{n=5}$ were calculated and are given in Table I.

The ratios of the theoretical values of $q_{n l m}$ show good agreement with the ratios of the experimental values of $V_{z z}$ in compounds of the same and of different oxidation states. This fact indicates that the effects of perturbing distortions on cubic symmetries are mainly responsible for the efg produced at the nucleus in chemical compounds of low oxidation states and that in these cases the ligand-field theory can be applied. The comparison also tells us that the efg acting on ${ }^{181} \mathrm{Ta}$ in these compounds are positive.

Thanks are due to Dr. J. D. Rogers for useful discussions and to CNPq (Brasil) for a research fellowship.

* This research was supported by the United States Air Force under Grant AF-AFOSR-1280-67, Conselho Nacional de Pesquisas (Brasil), and Conselho de Pesquisas da UFRGS (Brasil).

${ }^{\prime} \mathrm{R}$. W. Sommerfeldt, T. W. Cannon, L. W. Coleman, and L. Schecter, Phys, Rev. 138, B763 (1965).

2 G. Marest, I. Berkes, G. Bougnot, and R. Bernard, Compt. Rend. 262, B367 (1966).

${ }^{3}$ P. da R. Andrade, A. Maciel, and J. D. Rogers, Phys. Rev. 159, 196 (1967).

4 B. N. Figgis, Introduction to Ligand Field Theory (Interscience Publishers, Inc., New York, 1966).

${ }_{5}^{5}$ J. E. Slater, Quantum Theory of Atomic Struchure (McGrawHill Book Co., New York, 1960), Vol. 2.

${ }^{6}$ F. A. Cotton and G. Wilkinson, Advanced Inorganic Chemisiry (Interscience Publishers, Inc., New York, 1966).

\section{Observation of Charge Exchange Xenon-Methane Mixtures by Ion- Cyclotron Double Resonance*}

\author{
Roger P. Clow and Jean H. Futreli $\dagger$ \\ Department of Chemistry, University of Utah, \\ Salt Lake City, Utah 84112 \\ (Received 29 January 1969)
}

One of the intriguing results in the literature of ionmolecule reactions has been the failure of Field and Franklin to observe charge exchange in a xenon-methane mixture at elevated pressure in a mass-spectrometer ion source. ${ }^{1}$ The ionization potential of methane has been determined recently in a photoionization study to be about $12.6 \mathrm{eV},{ }^{2}$ while the recombination energies of the ${ }^{2} P_{3 / 2}$ and ${ }^{2} P_{1 / 2}$ states of $\mathrm{Xe}^{+}$are 12.13 and 13.44
$\mathrm{eV},{ }^{3}$ respectively. Thus charge exchange of the ${ }^{2} P_{1 / 2}$ state with $\mathrm{CH}_{4}$ to produce the methane molecular ion is clearly exothermic. Furthermore, this reaction has been observed in tandem mass-spectrometer experiments using accelerated and mass-selected $\mathrm{Xe}^{+4-7}$ and in single-source mass-spectrometer experiments using pulse methods $s^{7,8}$ or other techniques $s^{9,10}$ to perturb the normal steady-state distribution of ions.

To explain their unexpected results Field and Franklin suggested that either charge exchange did not occur between thermal velocity $\mathrm{Xe}^{+}$and methane molecules or that no net charge exchange occurs, the charge exchange between $\mathrm{Xe}^{+}\left({ }^{2} P_{1 / 2}\right)$ and methane being exactly balanced by charge exchange of $\mathrm{CH}_{4}{ }^{+}$with xenon to produce $\mathrm{Xe}^{+}\left({ }^{2} P_{3 / 2}\right)$. The recombination energies of relaxed $\mathrm{CH}_{4}{ }^{+}$range from 9 to $12 \mathrm{eV}$, ${ }^{11}$ so the latter explanation is plausible. Using ion-cyclotron double resonance, ${ }^{22}$ King and Elleman recently presented evidence that this balance of reactions occurs. ${ }^{13}$ Their results also indicated that the charge-exchange reaction between $\mathrm{CH}_{3}{ }^{+}$and $\mathrm{Xe}$ occurs, although it must be endothermic by $2.29 \mathrm{eV}$ for ground-state ions $\mathrm{s}^{3,24}$ and by at least $1.5 \mathrm{eV}$ for the distribution of ion energies formed by electron impact.".

For several months we have been studying ionmolecule reactions by ion-cyclotron resonance techniques for several systems whose reactions have been well characterized by established techniques. In most cases there has been at least qualitative agreement with earlier results, but in a few cases we have found clearcut contradictions. These appear to be attributable to two artifacts in ion-cyclotron resonance experiments, the extreme sensitivity to space charge (because of ion trapping in the strong magnetic field for lowenergy ions) and ill-defined ion losses with low and moderate irradiating fields (i.e., "leakiness" of the ICR cell because actual electrostatic fields deviate substantially from the idealized fields assumed in calculations). It occurred to us that these phenomena may have been involved in the charge exchange study, and we have reinvestigated the xenon-methane system.

In our experiments a 3:1 mixture of methane and xenon was investigated by ion-cyclotron resonance using various irradiation schemes. The results are displayed in Fig. 1. Figures 1(a) and 1(b) are double-resonance spectra ${ }^{12}$ in which $\mathrm{CH}_{4}{ }^{+}$is observed while the irradiating oscillator is swept over the $\mathrm{Xe}^{+}$resonance region, and ${ }^{129} \mathrm{Xe}^{+}$is observed while the irradiating oscillator is swept over the resonance frequencies of $\mathrm{CH}_{3}{ }^{+}$and $\mathrm{CH}_{4}{ }^{+}$, respectively. Figure 1 (b) is substantially equivalent to King and Elleman's Fig. $1 .^{13}$

Because ion-cyclotron resonance peaks are so sharp in a properly tuned system, and because a shift in space charge (as by irradiating ions out of the cavity in a double-resonance experiment) can shift the resonant frequency by a few gauss, erroneous signals are sometimes generated. Accordingly, we find it desirable 